\title{
ARTICLES
}

\section{EFFECT OF YOGA ON HYPERTENSION: REVIEW OF RELATED LITERATURE}

\author{
Mrs. Florine Clara Fernandes* \& Dr. Sikandar Kumar**
}

*Research Scholar, Himalayan University, Itanagar, Arunachal Pradesh, India.

**Research Guide, Himalayan University, Itanagar, Arunachal Pradesh, India. DOI: http://doi.org/10.47211/trr.2019.v05i02.014

Received $25^{\text {th }}$ November 2019, Accepted $5^{\text {th }}$ December 2019, Published $20^{\text {th }}$ December 2019

\section{ABSTRACT:}

Hypertension is a condition that can lead to heart strokes, and even death. It happens when the force of the blood pumping through our arteries is too strong. When our heart beats, it pushes blood through your arteries to the rest of your body. Yoga practices have been shown to be effective in reducing BP in hypertensive population. The probable mechanism by which yoga reduces BP is by reduction in sympathetic activity. These research review helped researchers understand Effect of Yoga on hypertension. More research is required to gain a better understanding of yoga impact on hypertension. It is important to conduct more studies on the results and reasons that how yoga impact on hypertension.

Key Words: Yoga, hypertension, high blood pressure.

\section{ABOUT AUTHORS}

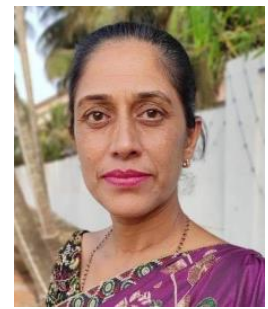

The author Mrs. Florine Clara Fernandes is research scholar, Himalayan University, Itanagar, Arunachal Pradesh, India.

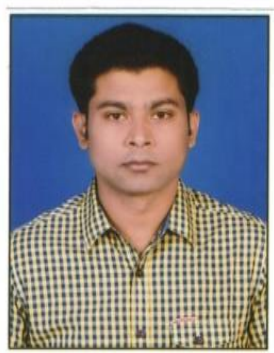

The author Dr. Sikandar Kumar has more than ten years of teaching experience. He has presented papers in various national and international conferences. 


\section{INTRODUCTION}

Review of literature is a systematic identification, location, scrutiny and summary of written material that contain information on research problem. The investigator, to gain insight and collect maximum information for building this study, carried out an extensive review of literature with the help of various sources.

\section{REVIEW OF LITERATURE}

Arpith, M.N. \& Devi, M.L. \& Manjunatha, S.N. (2019). This study is undertaken to see whether practice of yoga can help in reducing hypertension which is an independent risk factor of coronary heart disease and also to check whether it can act as an adjunct to pharmacy therapeutic treatment in mild to moderate hypertension. The present study was conducted among 80 hypertensive patients trained in yoga at S-VYASA, Mysuru and 80 control groups of hypertensive patients not practicing yoga attending Department of cardiology, KR Hospital, Mysuru. The parameters assessed were Pulse Rate, Systolic Blood Pressure, Diastolic Blood Pressure and Pulse Pressure. They were measured at baseline (before start of yoga), at 12 weeks and 24 weeks of intervals. The data was analyzed using paired and unpaired t test. Pulse rate, Systolic blood pressure, Diastolic blood pressure and pulse pressure were observed between yoga and control group. Statistically significant reduction were observed in Pulse Rate, Systolic Blood Pressure, Diastolic Blood Pressure and Pulse Pressure among yoga practitioners $(p<0.001)$. These changes in blood pressure are probably due to reduced stress and decreased sympathetic activity attributable to yoga.

Khan, Asma \& Rehman, AshfaqueUr \& Zaidi, Adil. (2018). Cardiovascular diseases (CVD) have emerges as the leading cause of mortality throughout the world. Essential hypertension is one of the important risk factor for CVD in modern society despite advanced and effective management. In this study, it has been observed that daily practice of yoga improves the blood pressure of mild hypertensive patients. Moreover, it substantially decreases the oxidative stress. Yoga can be an effective alternative to reduce the blood pressure in mild hypertensive patients either with medication or without medication.

Tiwari, Sunita \& Pal, Arvind. (2017). High blood pressure (BP) is a major public health problem worldwide. It is an important risk factor for acute myocardial infarction, cerebrovascular disease, chronic renal failure, and congestive heart failure. Lifestyle modifications play an important role in pre hypertensive individuals and also act as an adjunct to antihypertensive therapy. Yoga practices have been shown to be effective in reducing BP in hypertensive population. The probable mechanism by which yoga reduces BP is by reduction in sympathetic activity, facilitating autonomic balance, which reduces chemoreceptor responses, and enhancing baroreflex sensitivity. Yoga is also effective as an adjunct therapy in reducing antihypertensive medication use. There is a need for recognition of yoga by our health system as a complementary therapy for treating hypertension (HPT).

Jeenath justin (2016), conducted a study on assess the effectiveness of yoga (alternate nostril breathing) on blood pressure among hypertensive clients. The research design adopted was Quasi Experimental design. The conceptual framework for this study was based on modified Ludwig Von Bertalanffys General System Theory. The study has been conducted in Unicare Hospital, Rajkot. Purposive sampling technique has been adopted to select the desired sample. The sample size was 40 (20 under Experimental group and 20 under Control group). As an intervention of 15 days alternate nostril breathing exercise was practiced for experimental group. The data was collected through sphygmomanometer. The collected data were analyzed by using both descriptive and inferential statistical methods. $-t \|$ test was used to assess the effectiveness alternate nostril breathing on blood pressure among hypertensive clients. The obtained - $t \|$ value for systolic blood pressure was 31.2 and for diastolic blood pressure was 29.8 which is greater than 15 tabulated $-t \|$ value 3.8 which was highly significant at 0.001 . The findings of the study revealed that the alternate nostril breathing helps in reducing in high blood pressure among hypertensive clients.

Singh, Abhishek et al., (2016). The Yoga is an art of developing mental and physical health of the body. The study was done on 30 hypertensive male patients undergoing the treatment since 3-17 years. The basal recording of cardiovascular parameters like Blood Pressure [Diastolic and systolic], Heart Rate, Pulse Pressure and Rate Pressure Product were taken. After that, they were given the training of yoga exercises like Shavasana and Pranayama by a trained teacher for every 6 days of 6 weeks. Then again the recordings of the cardiovascular parameters were taken. It was found that there was a statistically significant decrease in all the cardiovascular parameters. The yoga exercise shows a strong tendency to improve the Autonomic Nervous System through enhanced parasympathetic nervous system and leading to decrease in the sympathetic tone in the skeletal muscle blood vessels. Thus by these results, it can be concluded that the yogic exercises can be treated as an alternative therapy for reducing stress disorders in persons suffering from essential hypertension. 
Satyanand, Vungarala et al (2016). Yoga is a remedy and highly effective in the treatment of high blood pressure. The present study aimed to evaluate the effectiveness of yoga in the treatment of high blood pressure. 100 subjects with complaints of high blood pressure attending the outpatient department of cardiology of Narayana medical college and hospital, Nellore are recruited into the study and after obtaining informed consent; yoga session was conducted in Narayana Yoga and Naturopathy Medical College and Hospital for a period of 12 weeks. The same number of age/sex matched control group with high blood pressure were also enrolled and kept without yoga techniques. During the period of treatment the symptom relief in the subjects is assessed periodically by a sphygmomanometer. Yoga is proved to be a best method to treat high blood pressure. Introduction:

Jayaram, Gadham \& Srikanth, Sajja \& Rooha, V. (2015). Hypertension is a major chronic lifestyle disease and an important public health problem worldwide. Mind-body therapies i.e., the transcendental meditation and Yoga have raised interest as they represent an alternative to medication; also contribute to an increased feeling of empowerment for patients in preventing and treating hypertension. Yoga, as a therapeutic regimen, has been shown to be useful to individuals with cardiovascular diseases and diabetes. The objective of our study is to study the effect of Pranayama and certain Yogic Asana's on BP, BMI \& lipid profile. Methods: 50 male subjects in the age group of 35-55 years were randomly selected based on exclusion criteria. BP, BMI and lipid profile was estimated before any intervention. They were subjected to pranayama and other Asana's for a period of 3 months. After 3 months of Yoga intervention, BP, BMI and lipid profile was estimated. Results: We observed a statistically significant decrease in Systolic BP, Diastolic BP and BMI after 3 months of Yoga training. Also, we observed a decrease in total cholesterol, VLDL, triglycerides and an increase in HDL cholesterol which is not statistically significant. Conclusion: A significant decrease in systolic blood pressure and diastolic blood pressure in subjects who have practiced Yoga, Asanas along with pranayama technique for 3 months duration.

Manchanda, Subhash \& Madan, Kushal. (2015). Hypertension is a major public health problem and is an important risk factor for stroke, coronary heart disease and renal failure. The blood pressure is not well controlled with drug therapy in large number of individuals, especially in the developing countries. There is a need for less expensive nonpharmacological alternative methods to control blood pressure. Yoga may be such cost-effective alternative. Several uncontrolled and randomized control trials have evaluated short- and long-term effects of yoga/meditation-based techniques in prehypertension and mild hypertension. Though, the results are mixed and there are several methodological limitations in reported studies, majority of studies demonstrate a modest decrease in both systolic and diastolic blood pressure. This modest decrease may significantly decrease the stroke mortality and coronary heart disease in general population. A recent scientific statement of American Heart Association also suggests that it is reasonable for all individuals with blood pressure levels more than $120 / 80 \mathrm{~mm}$ $\mathrm{Hg}$ to consider alternative approaches like transcendental meditation as adjunct method to lower blood pressure.

Cohen, Debbie. (2013). In his research found that there is a genuine need for rigorously conducted randomized clinical trials of yoga assessing the effects of lowering BP in patients with prehypertension and stage 1 hypertension. Even if the effects of yoga on hypertension are modest this can still provide substantial CV protection for this group of patients with mild to moderate hypertension and may afford patients the opportunity to engage in yoga instead of committing to lifelong antihypertensive medication.

Ranjana Ganpat (2013) conducted a quasi-experimental study on effectiveness of pranayama on blood pressure level. 60 hypertensive clients selected in this study. A selected intervention includes pranayama was given morning and evening in 10 days for 30 minutes. The post test was done in after 10th day. The blood pressure level before and after Pranayama shows highly significant difference between blood pressure from day 1, day 8, day 9 and day 10. Systolic blood pressure from $154.53 \mathrm{mmHg}$ (SD 7.53) to $133.2 \mathrm{mmHg}$ (SD 6.14) and diastolic blood pressure from $93.8 \mathrm{mmHg}$ (SD 4.99) to $82.26 \mathrm{mmHg}$ (SD 5.24). Paired t-test for blood pressure before and after Pranayama shows $p$ value 0.000000034 , which very less than 0.01 , therefore the effect of Pranayama on blood pressure of hypertensive samples is highly significant. The mean blood pressure on day 1 and day 10 shows the difference in systolic blood pressure was $21.33 \mathrm{mmHg}$ and diastolic blood pressure was $11.54 \mathrm{mmHg}$. The severity of blood pressure before and after Pranayama was seen. Before Pranayama moderate blood pressure is $71.66 \%$, mild is $19.99 \%$ and severe $8.33 \%$ and after Pranayama mild blood pressure is $54.99 \%$, normal $36.66 \%$ and moderate is $4.99 \%$. The severity of blood pressure before and after Pranayama in reduction of blood pressure. This study revealed that yoga can reduce blood pressure level.

Hagins, Marshall \& States, Rebecca \& Selfe, Terry \& Innes, Kim. (2013). To systematically review and metaanalyze the effectiveness of yoga for reducing blood pressure in adults with hypertension and to assess the modifying influences of type and length of yoga intervention and type of comparison group. Yoga can be 
preliminarily recommended as an effective intervention for reducing blood pressure. Additional rigorous controlled trials are warranted to further investigate the potential benefits of yoga.

Sao, Arun Kumar. (2011). evaluate effects of nada yoga on hypertension (SBP \& DBP) and anxiety level of subject. Sixty subjects in the age group of 25-35 years volunteered for the study. They were randomly divided in two groups of 30 each. Group 1 subjects served as controls and group 2 subjects practiced nada yoga for 30 minutes during the evening, for one month. Orthostatic blood pressure and anxiety were measured before and after one month of nada yoga practices. There was significant difference in the systolic blood pressure, diastolic blood pressure and the anxiety level between the control group and the experimental group. These observations suggest that nada yoga can be used as psycho-physiologic stimuli to improvement of blood pressure and anxiety, which, in turn, might be responsible for improved sense of well-being. Key words: - Nada yoga, hypertension, systolic and diastolic blood pressure, anxiety.

\section{CONCLUSIONS:}

The purpose of this review was to view the literature related to yoga on hypertension. It is clear from the research reviewed that it is required to develop yoga based knowledge on patients of high blood pressure. It revealed that extensive research is needed to understand the benefits of yoga for hypersensitive patients.

\section{REFERENCES:}

1. Arpith, M.N. \& Devi, M.L. \& Manjunatha, S.N. (2019). Effect of Yoga on Mild to Moderate Hypertension. International Journal of Physiology. 7. 35. 10.5958/2320-608X.2019.00133.1.

2. Cohen, Debbie. (2013). Yoga and Hypertension. Journal of Yoga \& Physical Therapy. 03. 10.4172/21577595.1000144.

3. Hagins, Marshall \& States, Rebecca \& Selfe, Terry \& Innes, Kim. (2013). Effectiveness of Yoga for Hypertension: Systematic Review and Meta-Analysis. Evidence-based complementary and alternative medicine: eCAM. 2013. 649836. 10.1155/2013/649836.

4. Jayaram, Gadham \& Srikanth, Sajja \& Rooha, V. (2015). Effect of Yoga on obesity, hypertension and lipid profile. International Journal of Research in Medical Sciences. 3. 1061. 10.5455/2320-6012.ijrms20150506.

5. Khan, Asma \& Rehman, AshfaqueUr \& Zaidi, Adil. (2018). Effects of Yoga on Blood Pressure and MDA in Mild Hypertensive Patients. Academia Journal of Medicine. 1. 10.21276/ajm.2018.1.2.1.

6. Manchanda, Subhash \& Madan, Kushal. (2015). Yoga and Hypertension. Hypertension Journal. 1. 28-33. 10.5005/jp-journals-10043-0005.

7. Ranjana Ganapat Ttryambake., Veena D. Sakhardande (2013). The effectiveness of Pranayama on blood pressure of hypertensive patients. International Journal of Sciences and Research, 4 (8), 561-564.

8. Satyanand, Vungarala \& Reddy, Bhakthavatsala \& Shaik, Mahaboob vali \& Mohanan, Dhivya \& Salma, Shaik \& Nuzhath, F. (2016). Effect of Yoga on Hypertension. Narayana Medical Journal. 5. 5-11.

9. Salivendra Dileep. \& Jeenath Justin Doss K. (2016). A Study to Assess the Effectiveness of Alternate Nostril Breathing on Blood Pressure among Hypertensive Clients in a Selected Hospital at Rajkot. Int. J. Adv. Nur. Management, 4(4), 369-371.

10. Sao, Arun Kumar. (2011). the Impact of Nada Yoga on Hypertension and Anxiety Level. 2. 15-22.

11. Singh, Abhishek \& Malhotra, Varun \& Tripathi, Yogesh \& Gupta, Shivani \& Dhar, Ushar \& Student, P. (2016). Beneficial effects of yoga on cardiovascular parameters in hypertensive patients. Santosh University Journal of Health Sciences. 2. 1-4.

12. Tiwari, Sunita \& Pal, Arvind. (2017). Yoga and Hypertension. Hypertension Journal. 3. 189-192. 10.5005/jpjournals-10043-0094. 